\title{
Pulmonary embolism response teams: A concept in progress and beyond borders
}

\author{
Gustavo A Heresi' ${ }^{1}$, Mateo Porres-Aguilar ${ }^{2}$ \\ 'Department of Pulmonary and Critical Care Medicine, Respiratory Institute, Cleveland Clinic, Cleveland, OH, USA \\ 2Department of Medicine, Division of Hospital and Adult Thrombosis Medicine, Texas Tech University Health Sciences Center and Paul L Foster School of Medicine, \\ EI Paso, TX, USA
}

\section{Related article}

by Araszkiewicz et al.

see p. 1311

Correspondence to: Gustavo A Heresi, MD, MS, Department of Pulmonary and Critical Care Medicine, Cleveland Clinic, 9500 Euclid Ave, Cleveland, OH 44195, USA, phone: +1 2166365327 e-mail: heresig@ccf.org

Copyright by the Author(s), 2021

Kardiol Pol. 2021;

79 (12): 1301-1302;

DOI: 10.33963/KP.a2021.0174

Received:

December 6, 2021

Accepted:

December 6, 2021

Published online:

December 7, 2021
Acute pulmonary embolism $(P E)$ remains a leading cause of death worldwide [1]. The care of PE patients, particularly those with intermediate and high-risk $P E$, is highly complex [2]. Indeed, the best treatment is unknown, and there is no standard approach. Historically, there has not been a well-organized, structured, systematic approach to acute PE management, with significant heterogeneity and inconsistencies in therapeutic decision-making. Options are myriad, including anticoagulation alone, systemic full dose or low dose thrombolysis, catheter directed low dose thrombolysis, catheter aspiration thrombectomy, surgical embolectomy, and insertion of a vena cava filter. These therapeutic options are performed by different specialists, without a single team coordinating and directing care. Practice varies significantly by location and resources. A small proportion of eligible PE patients receive advanced reperfusion therapies. Reasons for this underutilization include failure to recognize potential benefit and integrate data in real time, inability to respond rapidly due to systems barriers, fear of complications and paralysis in decision-making in the setting of life-threatening situations [3].

The concept of a single $P E$ response team (PERT) emerged as a response to this status quo. While the composition and operations of these teams vary across institutions, there are some key characteristics that a PERT should have. A PERT is a multidisciplinary team of experts in the care of $\mathrm{PE}$, with a centralized unique activation process, that can provide rapid assessment and risk stratification of the PE patient, formulate an individualized treatment approach, and facilitate the implementation of the recommended therapeutic strategy $[3,4]$. The PERT concept was initially established in the USA in 2012 at the Massachusetts General Hospital (MGH) [5]. The concept disseminated rapidly in the USA, leading to the formation of the PERT consortium [6]. Now, the PERT concept is going global.

In this context, the study by Araszkiewicz et al. [7] in the current issue of Kardiologia Polska (Kardiol Pol, Polish Heart Journal) represents a welcomed addition to the PERT literature. The authors present the polish PERT initiative, by describing the first national, multicenter analysis of patients cared for by four PERTs between 2018 and 2020. This is the first report of this nature from Europe. A total of 680 PERT activations occurred during the study period. Interestingly, $47 \%$ of PERT activations were not related to high-risk nor intermediate-high risk PE patients; however, $82 \%$ of patients had central PE, and moreover, $81.3 \%$ of the patients categorized as low-risk PE had central PE. A recent report also identified large clot burden as a trigger for PERT activation [8]. Almost one quarter of the study patients received at least one advanced therapy (e.g., catheter directed therapies, systemic thrombolysis, inferior vena cava filter insertion), with significant variability among the four institutions, perhaps related to differences in local availability of resources and expertise. There was also a significant difference in in-hospital mortality rates among the four 
institutions, with an overall in-patient mortality of 5.1\%, and PE-related mortality of $2.8 \%$. These rates are similar to the PERT experience in the USA $[9,10]$. Furthermore, recent retrospective [10] and prospective data [9] have shown that the implementation of a PERT in an institution is associated with an increased utilization of advanced PE therapies, as well as improved outcomes. Of note, this is achieved even when the PERT is not actively involved in all cases reported $[9,10]$, suggesting that there are benefits from simply having a functioning PERT in an institution, likely related to increased PE awareness and education after PERT implementation.

Araszkiewicz et al. [7] report the use of systemic anticoagulation alone in $80 \%$ of PERT activations, higher rates than prior retrospective reports $[5,11]$, but consistent with the notion that most acute PE patients cared for by PERT teams do well with anticoagulation alone. While the implementation of PERT teams is associated with an increased use of advanced PE therapies, these invasive and riskier strategies should not be used liberally, and are best utilized in experienced operators' hands, depending on the local resources and expertise, and in the context of an individualized treatment plan. For example, completely occlusive acute PE disease in an unstable patient might be best treated with a catheter based procedure, as systemically infused thrombolytics are unlikely to be effective [12]. The ongoing PEITHO-3 and HI-PEITHO randomized trials will hopefully elucidate the best patients' clinical phenotypes for the use of low-dose systemic thrombolysis and ultrasound facilitated, catheter-directed thrombolysis, respectively $[13,14]$.

The availability of a PERT allows clinicians to balance the pros and cons of advanced or invasive therapeutic maneuvers for a given patient with acute PE. Of course, such teams, as defined above, are challenging to implement and can impose a burden on participating specialists. Different institutions should tailor specifics of PERT logistics to leverage their local expertise and resources. Emerging data point to the fact that the multidisciplinary approach of PERT leads to improved outcomes and safety. In that context, we are pleased to see the PERT concept go global. However, much remains to be learned about the composition, operations, logistics and impact of PERTs across the world. We need more reports such as the excellent work presented here.

\section{Article information}

Conflict of interest: None declared.

Open access: This article is available in open access under Creative Common Attribution-Non-Commercial-No Derivatives 4.0 International (CC BY-NC-ND 4.0) license, allowing to download articles and share them with others as long as they credit the authors and the publisher, but without permission to change them in any way or use them commercially. For commercial use, please contact the journal office at kardiologiapolska@ptkardio.pl.

How to cite: Heresi GA, Porres-Aguilar M. Pulmonary embolism response teams: A concept in progress and beyond borders. Kardiol Pol. 2021; 79(12): 1301-1302, doi: 10.33963/KP.a2021.0174.

\section{REFERENCES}

1. Khan F, Tritschler T, Kahn S, et al. Venous thromboembolism. Lancet. 2021; 398(10294): 64-77, doi: 10.1016/s0140-6736(20)32658-1, indexed in Pubmed: 33984268.

2. Konstantinides SV, Meyer G, Becattini C, et al. 2019ESC Guidelines for the diagnosis and management of acute pulmonary embolism developed in collaboration with the European Respiratory Society (ERS): The Task Force for the diagnosis and management of acute pulmonary embolism of the European Society of Cardiology (ESC). Eur Respir J. 2019; 54(3): 543-603, doi: 10.1183/13993003.01647-2019, indexed in Pubmed: 31473594.

3. Serhal $M$, Haddadin IS, Heresi GA, et al. Pulmonary embolism response teams. J Thromb Thrombolysis. 2017; 44(1): 19-29, doi: 10.1007/s11239017-1498-9, indexed in Pubmed: 28401327.

4. Porres-Aguilar M, Anaya-Ayala JE, Heresi GA, et al. Pulmonary embolism response teams: a novel approach for the care of complex patients with pulmonary embolism. Clin Appl Thromb Hemost. 2018;24(9_suppl):48S-55S, doi: 10.1177/1076029618812954, indexed in Pubmed: 30453745.

5. Kabrhel C, Rosovsky R, Channick R, et al. A multidisciplinary pulmonary embolism response team: initial 30-month experience with a novel approach to delivery of care to patients with submassive and massive pulmonary embolism. Chest. 2016; 150(2):384-393, doi: 10.1016/j.chest.2016.03.011, indexed in Pubmed: 27006156.

6. Schultz J, Giordano N, Zheng $\mathrm{H}$, et al. A multidisciplinary pulmonary embolism response team (PERT) - experience from a national multicenter consortium. Pulm Circ. 2019; 9(3): 204589401882456, doi: 10.1177/2045894018824563, indexed in Pubmed: 30632901.

7. Araszkiewicz A, Kurzyna M, Kopeć G, et al. Pulmonary embolism response team - a multidisciplinary approach to pulmonary embolism treatment. Polish PERT initiative report. Kardiol Pol. 2021; 79(12): 1311-1319, doi: 10.33963/KP.a2021.0130, indexed in Pubmed: 34643260.

8. Mortensen CS, Kramer A, Schultz JG, et al. Predicting factors for pulmonary embolism response team activation in a general pulmonary embolism population. J Thromb Thrombolysis. 2021 [Epub ahead of print], doi: 10.1007/s11239-021-02533-0, indexed in Pubmed: 34370168.

9. Lacey M, Hammad T, Li J, et al. Prospective experience of pulmonary embolism management and outcomes. J Invasive Cardiol. 2021; 33(3): E173-E180, indexed in Pubmed: 33570502.

10. Chaudhury P, Gadre SK, Schneider E, et al. Impact of multidisciplinary pulmonary embolism response team availability on management and outcomes. Am J Cardiol. 2019; 124(9): 1465-1469, doi: 10.1016/j.amjcard.2019.07.043, indexed in Pubmed: 31495443.

11. Mahar JH, Haddadin I, Sadana D, et al. A pulmonary embolism response team (PERT) approach: initial experience from the Cleveland Clinic. J Thromb Thrombolysis. 2018; 46(2): 186-192, doi: 10.1007/s11239-0181686-2, indexed in Pubmed: 29855780.

12. Uflacker R. Interventional therapy for pulmonary embolism. J Vasc Interv Radiol. 2001; 12(2): 147-164, doi: 10.1016/s1051-0443(07)61821-1, indexed in Pubmed: 11265879.

13. Sanchez $\mathrm{O}$, Charles-Nelson A, Ageno W, et al. Reduced-Dose intravenous thrombolysis for acute intermediate-high-risk pulmonary embolism: rationale and design of the pulmonary embolism international thrombolysis (PEITHO)-3 trial. Thromb Haemost. 2021 [Epub ahead of print], doi: 10.1055/a-1653-4699, indexed in Pubmed: 34560806.

14. Ultrasound-facilitated, Catheter-directed, Thrombolysis in Intermediate-high Risk Pulmonary Embolism (HI-PEITHO). Available online: https://clinicaltrials.gov/ct2/show/NCT04790370 (accessed November 3, 2021). 